\title{
ERRATA, VOLUME 55
}

Becker, H. W., $A$ new proof and application of Kirchoff's rules. Bull. Amer. Math. Soc. Abstract 55-7-437.

P. 718, lines 5 and 6 of the abstract. For "modes" read "nodes," by which was meant cut-sets of branches meeting at the same nodes.

\section{ERRATA, VOLUME 57} tions.

Bourgin, D. G. Classes of transformations and bordering transforma-

p. 223. Following equation (1) add: "More generally, with $S$ on $X$ to $Z, T$ on $X$ to $Y$, and $R$ on $Y$ to $Z$,

$$
\left(S\left(x_{1} \bigcirc x_{2}\right), R\left(T x_{1} \oplus T x_{2}\right)\right) \subset \mathfrak{M} . "
$$

p. 223, line 6 from the bottom, equation (2), and line 4 from the bottom. For " $\mathfrak{R}^{\prime}$ " read " $\mathfrak{M}$ "."

p. 224, line 23 . For " $S$ is the identity operator" read " $Z$ is the real axis."

p. 224, line 25. For "( 1 ) is" read " $\left(1^{\prime}\right)$ is (with $\left.S=R=\|\|\right)$."

p. 225 , line 12 . For " $<\infty$ " read " $=0$."

p. 225, lines 21 and 22. For "studied $U(x)$ turns out to be" read " $U(x)$ is."

p. 233, line 15. For "neighborhood retract which is" read "neighborhood retract or LC* space which is."

Brahana, H. R., George Abram Miller, 1863-1951.

p. 382, line 5. For "1901" read "1909."

Stoker, J. J. Review of Hydrodynamics, a study in logic, fact, and similitude, by G. Birkhoff.

p. 497, line 6 from the bottom. For "treats" read "emphasizes."

p. 498, line 8. For "not mentioned" read "insufficiently stressed."

p. 498, line 19. After "differential equations" add "(as was mentioned earlier in the same chapter by the author)."

\section{ERRATA, VOLUME 58}

Becker, H. W., Pythagorean tetrahedrons, hexahedrons, and graphs. Bull. Amer. Math. Soc. Abstract 58-5-510.

p. 561. For the next to the last sentence of the abstract read "But a P.H. is geometrically a hexahedron, pentahedron, or tetrahedron (due to faces lying in the same plane) according as the common face contains the longest edge of one or both component tetrahedrons, or neither. For the latter case, see H. S. M. Coxeter, Regular polytopes, 1947, p. 71." 